\title{
MicroRNA-138-5p drives the progression of heart failure via inhibiting sirtuin 1 signaling
}

\author{
SHUAI SUN, CHUN WANG and JIANXIN WENG \\ Department of Cardiology, Peking University Shenzhen Hospital, Shenzhen, Guangdong 518000, P.R. China
}

Received July 13, 2020; Accepted November 27, 2020

DOI: $10.3892 / \mathrm{mmr} .2021 .11915$

\begin{abstract}
The present study aimed to investigate the regulatory effects of microRNA-138-5p (miR-138-5p) and sirtuin 1 (SIRT1) on the progression of heart failure (HF). The binding association between miR-138-5p and SIRT1 was assessed by the dual-luciferase reporter assay. By conducting reverse transcription-quantitative polymerase chain reaction and Western blotting, relative levels of SIRT1 and p53 regulated by miR-138-5p were detected. In vitro HF models were generated by hydrogen peroxide $\left(\mathrm{H}_{2} \mathrm{O}_{2}\right)$ induction in $\mathrm{AC}-16$ and human cardiomyocyte (HCM) cells, followed by detection of the regulatory effects of SIRT1 on cell apoptosis and p53 expression. MiR-138-5p was negatively correlated with the SIRT1 level in cardiomyocytes. By recognizing and specifically targeting SIRT1 3'-untranslated region (3'-UTR), miR-138-5p decreased the translational level of SIRT1 and inhibited its enzyme activity, thereby decreasing the deacetylation level of p53. Through downregulating SIRT1 and activating p53 signaling, miR-138-5p induced apoptosis in $\mathrm{H}_{2} \mathrm{O}_{2}$-induced AC-16 and HCM cells. By contrast, knockdown of miR-138-5p in the in vitro HF models significantly protected the cardiomyocytes. SIRT1 contributed toward alleviate HF by inhibiting cardiomyocyte apoptosis via enhancing the deacetylation level of p53. MiR-138-5p decreases the enzyme activity of SIRT1 by specifically targeting its 3'-UTR and activates p53 signaling, followed by triggering cardiomyocyte apoptosis during the process of HF. It is considered that miR-138-5p and SIRT1 may be potential diagnostic biomarkers and therapeutic targets for $\mathrm{HF}$.
\end{abstract}

\section{Introduction}

Being a common outcome of numerous cardiovascular diseases, heart failure (HF) is characterized by high incidence

Correspondence to: Professor Shuai Sun, Department of Cardiology, Peking University Shenzhen Hospital, 1120 Lianhua Road, Shenzhen, Guangdong 518000, P.R. China

E-mail: 358772811@qq.com

Key words: microRNA-138-5p, sirtuin 1, p53, acetylation, heart failure and poor prognosis, and it is a serious health problem worldwide $(1,2)$. Myocardial infarction is the main cause of HF. A series of pathological lesions following myocardial infarction, including cardiomyocyte apoptosis, inflammatory response and overactivation of the neurohumoral system, result in ventricular remodeling and thus HF (3). Although progress has been made in the fundamental research of HF following myocardial infarction, a large number of myocardial infarction patients are not diagnosed and treated in a timely manner owing to atypical symptoms. They eventually deteriorate to $\mathrm{HF}$ or arrhythmia (4). Therefore, improvement of the diagnostic rate in the early phase and investigation of relevant regulatory targets are necessary to enhance the therapeutic efficacy and prognosis in patients with HF or myocardial infarction.

Sirtuin 1 (SIRT1) is a highly conserved NAD-dependent histone deacetylase, which is abundantly expressed in mammalian hearts. SIRT1 serves a vital role in regulating the energy metabolism of cardiac muscle cells, production of reactive oxygen species (ROS), induction of angiogenesis and inhibition of autophagy $(5,6)$. It is reported that SIRT1 exerts a protective effect in HF by alleviating oxidative stress, fibrosis and inflammation through SIRT1/PCG-1 $\alpha$ signaling (7). Previous studies have demonstrated that microRNA-138-5p (miR-138-5p) is able to downregulate SIRT1, its downstream target. The inhibitory role of miR-138-5p in tumor progression and metastasis has been identified $(8,9)$. Recently, experimental studies have demonstrated that miR-138-5p is involved in the process of heart diseases $(10,11)$. Wang et al $(10)$ proposed that overexpression of miR-138-5p leads to aggravation of cardiac injury through accelerating cardiac hypoxia and reperfusion via inactivating the SIRT1-PGC-1 $\alpha$ axis. However, the potential function of miR-138-5p in the progression of HF remains largely unclear.

During the progression of HF, cardiomyocyte apoptosis is of great significance. Due to the non-renewability of the myocardium, apoptotic or necrotic cardiomyocytes are recognized by the body and replaced by scar tissue. Subsequently, insufficient blood supply and ventricular remodeling ultimately lead to $\mathrm{HF}$ (12). $\mathrm{A} \mathrm{H}_{2} \mathrm{O}_{2}$-induced $\mathrm{HF}$ model was widely used in in vitro or in vivo $\mathrm{HF}$ research $(13,14)$. In the present study, in vitro $\mathrm{HF}$ models were generated by $\mathrm{H}_{2} \mathrm{O}_{2}$ induction in $\mathrm{AC}-16$ and human cardiomyocyte (HCM) cells. The role of miR-138-5p in influencing the process of HF and the involvement of SIRT1 were mainly investigated. 


\section{Materials and methods}

Cell culture. Human cardiomyocyte cell lines, including AC16 (cat. no. BNCC337712), HCM (cat. no. BNCC337719), HCFB (cat. no. BNCC339420) and CCC-HEH-2 (cat. no. BNCC100615) were obtained from the BeNa culture collection and cardiomyocyte HCM-a cells (cat. no. XK-1262) were purchased from Shanghai Xuanke Biotechnology Co., Ltd. Cells were cultured in Dulbecco's modified Eagle's medium (DMEM; Gibco; Thermo Fisher Scientific, Inc.) containing $10 \%$ fetal bovine serum (FBS; Gibco; Thermo Fisher Scientific, Inc.), and $1 \%$ penicillin and streptomycin in a humidified atmosphere of $5 \% \mathrm{CO}_{2}$ at $37^{\circ} \mathrm{C}$. Cell passage was conducted using trypsin until adherent cells were grown to $>80 \%$ confluence.

Dual-luciferase reporter assay. Binding sites were predicted between the seed sequences of miR-138-5p and SIRT1 using TargetScan 7.2 (http://www.targetscan.org/vert_72). Sequences containing the SIRT1 3'-untranslated region (3'-UTR), mutant SIRT1 3'-UTR and miR-138-5p sequences were constructed by CoBioer Biosciences Co., Ltd. DNAs were directly synthesized by annealing, and luciferase vectors were constructed using pmirGLO (Promega Corporation) following splicing by the restriction endonucleases NheI and SalI. Plasmids were extracted following culturing with E. coli, and they were respectively named as pmirGLO-SIRT1-3'UTR and pmirGLO-SIRT1-mut3'UTR. A plasmid overexpressing miR-138-5p was generated using pcDNA3.1(+), which was named as pcDNA3.1(+)-miR-138-5p.

AC-16 cells were transfected with plasmids classified as follows: i) pmirGLO-SIRT1-3'UTR + pcDNA3.1(+); ii) pmirGLO-SIRT1-3'UTR + pcDNA3.1(+)-miR-138-5p; iii) pmirGLO-SIRT1-mut3'UTR + pcDNA3.1(+;) and iv) pmirGLO-SIRT1-mut3'UTR + pcDNA3.1(+)-miR-138-5p In brief, AC-16 cells were seeded onto a 96-well plate and cultured to $\sim 80 \%$ confluence. Cell transfection was performed using Lipofectamine 2000 (Invitrogen; Thermo Fisher Scientific, Inc.) for $6 \mathrm{~h}$, then the medium was replaced with fresh culture medium. At $48 \mathrm{~h}$ post-transfection, $100 \mu \mathrm{l}$ cell lysate was added to each well, and the mixture was collected and centrifuged at room temperature at $13,500 \mathrm{x} \mathrm{g}$ for $5 \mathrm{~min}$. A total of $50 \mu 1$ supernatant was incubated in $100 \mu 1$ Firefly luciferase detection reagent (Promega Corporation). They were rapidly mixed together and subjected to detection of relative light units (RLU). Three replicates were set in each group. Relative firefly luciferase activity was normalized to Renilla luciferase activity.

Cell transfection. Oligonucleotide sequences of the miR-138-5p mimic (5'-AGCUGGUGUUGUGAAUCAGGCCG-3') and miR-138-5p inhibitor (5'-CGGCCUGAUUCACAACACCAG CU-3'), as well as their negative controls (NC; miR-138-5p mimic-NC, 5'-UUCUCCGAACGUGUCACGUTT-3'; and miR-138-5p inhibitor-NC, 5'-UUCUCCGAACGUGUCACG UTT-3') were purchased from Guangzhou RiboBio Co., Ltd. All oligonucleotides were transfected at a final concentration of $50 \mathrm{nM}$. Cell transfection was performed using Lipofectamine 2000 (Invitrogen; Thermo Fisher Scientific, Inc.). Briefly, AC-16 and HCM cells were seeded onto a 24-well plate and cultured for $24 \mathrm{~h}$. Until cell confluence reached $>70 \%$, cells were cultured in serum-free DMEM for $4 \mathrm{~h}$. miR-138-5p mimic, miR-138-5p inhibitor or NC was respectively mixed with polyethylenimine at room temperature for $40 \mathrm{~min}$, followed by application of serum-free Opti-MEM (Gibco; Thermo Fisher Scientific, Inc.). Cells were incubated in the transfection mixture at a final concentration of $100 \mathrm{nM}$ at $37^{\circ} \mathrm{C}$ in a humidified atmosphere containing $5 \% \mathrm{CO}_{2}$ for $6 \mathrm{~h}$, and then the transfection mixture was replaced with DMEM containing $10 \%$ FBS, and $1 \%$ penicillin and streptomycin. Transfection efficacy was observed under a fluorescence microscope (magnification, x100) using FAM-labeled miRNAs as internal references. Cells were used following 24-48 h of transfection for subsequent experiments.

Generation of the in vitro HF models. AC-16 and HCM cells transfected for $24 \mathrm{~h}$ in the logarithmic growth phase were prepared in suspension at $1 \times 10^{4}$ cells $/ \mathrm{ml}$. Cell suspension was applied to a 6-well plate with $2 \mathrm{ml} /$ well. Following culturing for $24 \mathrm{~h}$, DMEM containing 2.5\% FBS was replaced for 12-h synchronization treatment. Subsequently, cells were induced in a medium containing or not containing $200 \mu \mathrm{M}$ hydrogen peroxide $\left(\mathrm{H}_{2} \mathrm{O}_{2}\right)$ for $6 \mathrm{~h}$. Successful generation of the in vitro $\mathrm{HF}$ models was defined through detecting the relative level of natriuretic peptide precursor B (NPPB) $(15,16)$.

Detection of ROS level. AC-16 and HCM cells were collected and inoculated into a 24-well plate. When the cell density was $\sim 80 \%, 10 \mu \mathrm{mol} / 1$ 2,7-Dichlorodi-hydrofluorescein diacetate was added into each well, and the cells were cultured at $37^{\circ} \mathrm{C}$ for $30 \mathrm{~min}$. Subsequently, five visual fields were randomly selected under the fluorescence microscope (magnification, $\mathrm{x} 100)$ to analyze the green fluorescence intensity, and the mean fluorescence intensity (MFI) was used to demonstrate the ROS level.

Reverse transcription-quantitative polymerase chain reaction (RT-qPCR). Cellular RNA was isolated by TRIzol ${ }^{\circledR}$ (Invitrogen; Thermo Fisher Scientific, Inc.). Then, cellular RNA was processed by gDNA eraser and reverse transcribed at $37^{\circ} \mathrm{C}$ for $15 \mathrm{~min}$ and $85^{\circ} \mathrm{C}$ for $5 \mathrm{sec}$ (maintained at $4^{\circ} \mathrm{C}$ ) into cDNA using a PrimeScript ${ }^{\mathrm{TM}}$ RT reagent kit with gDNA Eraser (Takara Bio, Inc.). qPCR was subsequently performed using a SYBR Premix Ex Taq ${ }^{\mathrm{TM}}$ II (Tli RNase H Plus) kit (Takara Bio, Inc.). qPCR was conducted at $95^{\circ} \mathrm{C}$ for $15 \mathrm{~min}$, followed by 40 cycles at $95^{\circ} \mathrm{C}$ for $5 \mathrm{sec}, 60^{\circ} \mathrm{C}$ for $30 \mathrm{sec}$ and $72^{\circ} \mathrm{C}$ for $40 \mathrm{sec}$, followed by a final extension at $72^{\circ} \mathrm{C}$ for $10 \mathrm{~min}$. Relative levels of NPPB, SIRT1, p53 and miR-138-5p were determined using the internal references of GAPDH and U6. Each experiment was conducted in triplicate. Primer sequences are listed in Table I. The data were quantified using the $2^{-\Delta \Delta \mathrm{Cq}}$ method (17).

Western blotting. Cardiomyocytes were transfected or induced with $\mathrm{H}_{2} \mathrm{O}_{2}$ as described earlier. Subsequently, protein levels of SIRT1, p53 and acetylated p53 were determined by western blotting. In brief, cells were lysed to isolate total proteins using RIPA lysis buffer (Beyotime Institute of Biotechnology), and total concentrations were determined using the bicinchoninic acid method. Protein samples (50 $\mu \mathrm{g}$ per lane) were subjected to $10 \%$ SDS-PAGE and transferred onto polyvinylidene 
Table I. Sequences of primers.

\begin{tabular}{ll}
\hline Genes & \multicolumn{1}{c}{ Sequences } \\
\hline miR-138-5p & 5'-AGCTGGTGTTGTGAATCAGG-3' \\
SIRT1 forward & 5'-GTATTTATGCTCGCCTTGCTG-3' \\
SIRT1 reverse & 5'-TGACAGAGAGATGGCTGGAA-3' \\
NPPB forward & 5'-AAGGAGGCACTGGGAGAGGGG \\
& AAT-3' \\
NPPB reverse & 5'-CCCCACCAAGCCAACACAGGAT \\
& GGA-3' \\
p53 forward & 5'-GAGCGAATCACGAGGGAC-3' \\
p53 reverse & 5'-GCACAAACACGGACAGGA-3' \\
GAPDH forward & 5'-AGCCACATCGCTCAGACAC-3' \\
GAPDH reverse & 5'-GCCCAATACGACCAAATCC-3' \\
U6 forward & 5'-CTCGCTTCGGCAGCACA-3' \\
U6 reverse & 5'-AACGCTTCACGAATTTGCGT-3'
\end{tabular}

miR, microRNA; NPPB, natriuretic peptide precursor B

fluoride (PVDF) membranes. Non-specific antigens in the PVDF membranes were blocked using 5\% skimmed milk at room temperature for $1 \mathrm{~h}$. Following washing in TBS- $0.05 \%$ Tween-20, the membranes were incubated with the following primary antibodies (dilution, 1:1,000; all ABclonal Biotech Co., Ltd.) at $4^{\circ} \mathrm{C}$ overnight: Anti-SIRT1 (cat. no. A19667), anti-p53 (cat. no. A19585), anti-acetyl-p53 (cat. no. A16324) and anti-GAPDH (cat. no. AC001). Following the primary antibody incubation, the membranes were incubated with the secondary antibody (dilution, 1:1,000; cat. no. AS014; ABclonal Biotech Co., Ltd.) at room temperature for $1 \mathrm{~h}$. Band exposure was achieved using enhanced chemiluminescence reagent (Sangon Biotech Co., Ltd.). Protein expression levels were quantified using Quantity One ${ }^{\circledR}$ 1-D Analysis software (version 4.6.8; Bio-Rad Laboratories, Inc.).

Flow cytometric analysis of cell apoptosis. Following $\mathrm{H}_{2} \mathrm{O}_{2}$ induction for $6 \mathrm{~h}$, cells were subjected to apoptosis detection using the Annexin V-FITC and propidium iodide (PI) Apoptosis kit (US Everbright Inc.). To begin with, cells were prepared in suspension at $5 \times 10^{5}$ cells $/ \mathrm{ml}$. Cell suspension was incubated with $5 \mu \mathrm{l}$ Annexin V-FITC and $5 \mu \mathrm{l}$ PI in the dark at room temperature for $15 \mathrm{~min}$, and subjected to flow cytometry (FACSCalibur; BD Biosciences). Apoptotic cells were analyzed using CellQuest Pro software (version 5.1; BD Biosciences). Annexin V-FITC-positive cells were considered to be apoptotic cells.

Determination of the enzymatic activity of SIRT1.SIRT1 Direct Fluorescent Screening assay kit (Abcam; cat. no. ab156915) was used to assess the enzyme activity of SIRT1. Total protein (30 $\mu \mathrm{g}$ per well in a 96 -well plate) was incubated with the reaction mixture containing buffer, SIRT1 substrate with fluorophore and quencher, and protease. Absorbances at $360 \mathrm{~nm}$ excitation wavelength and $465 \mathrm{~nm}$ emission wavelength were recorded using the microplate reader (BioTek Instruments, Inc.).
Lentivirus transfection. A lentivirus overexpressing SIRT1 (GV287-SIRT1) was constructed by cleavage of GV287 plasmid (Shanghai GeneChem Co., Ltd.) with AgeI and thus splicing SIRT1. A 2nd generation system was used to package the lentivirus. The lentiviral plasmid, packaging vector and envelope vector were mixed at a 4:3:2 ratio for a total DNA mass of $20 \mu \mathrm{g}$. In brief, 293T cells were cultured to $80 \%$ confluence and then DMEM was replaced by Opti-MEM. Following cell culture for $4 \mathrm{~h}$ at $37^{\circ} \mathrm{C}$, cells were incubated with Lenti-Easy Packaging mix and GV287-SIRT1 (or GV287 as negative control) for $5 \mathrm{~min}$ at room temperature, then Lipofectamine for a further $20 \mathrm{~min}$, prior to the addition of 293T fresh culture medium (cat. no. BNCC352005; BeNa Culture Collection; Beijing Beina Chunglian Biotechnology Research Institute) for $6 \mathrm{~h}$ at $37^{\circ} \mathrm{C}$. Following the incubation, the medium was replaced with DMEM containing $10 \% \mathrm{FBS}, 1 \%$ penicillin and streptomycin and cultured to day 3. The supernatant of transfected $293 \mathrm{~T}$ cells was collected at day 3, which was filtered for detecting viral titers. Lentiviral transfection (at a multiplicity of infection of 5) in AC-16 and HCM cells was conducted for $12 \mathrm{~h}$ at $37^{\circ} \mathrm{C}$ when cell confluence reached $\sim 80 \%$. Fluorescence expression was observed 3 days later.

Statistical analysis. Statistical analyses were performed using GraphPad Prism 8 (GraphPad Software, Inc.). Data are expressed as the mean \pm standard deviation. All data conformed to a normal distribution. Comparisons among multiple groups were analyzed using one-way analysis of variance followed by Tukey's or Bonferroni's post hoc test. The correlation analysis was performed by Pearson's correlation analysis. $\mathrm{P}<0.05$ was considered to indicate a statistically significant difference. All experiments were performed in triplicate and repeated three times.

\section{Results}

A negative correlation between SIRT1 and miR-138-5p in cardiomyocytes. Following cell culture in DMEM containing $10 \%$ FBS for $48 \mathrm{~h}$, the relative levels of SIRT1 and miR-138-5p were detected in cardiomyocyte cell lines (AC16, HCM, HCM-a, HCFB and CCC-HEH-2) by RT-qPCR and Western blotting. Relative RNA expression is presented in Fig. 1A and B, and protein expression is presented in Fig. 1C. According to the Pearson's correlation analysis, a negative correlation was identified between SIRT1 and miR-138-5p at mRNA levels.

miR-138-5p targets SIRT1. Binding sites were predicted by TargetScan and the result was shown in Fig. 2A. The dual-luciferase reporter assay revealed that luciferase intensity in cells co-transfected with pmirGLO-SIRT1-3'UTR and pcDNA3.1(+)-miR-138-5p was $48 \%$ of that in the control group $(\mathrm{P}<0.01)$. Notably, luciferase intensity in cells co-transfected with pmirGLO-SIRT1-mut3'UTR and pcDNA3.1(+)-miR-138-5p was up to $99 \%$ of that in the control group, demonstrating no significant difference $(\mathrm{P}>0.05$; Fig. 2B). This indicated that miR-138-5p may regulate SIRT1 with sequence specificity.

miR-138-5p inhibits the expression level and enzyme activity of SIRT1. The respective expression level and enzymatic 

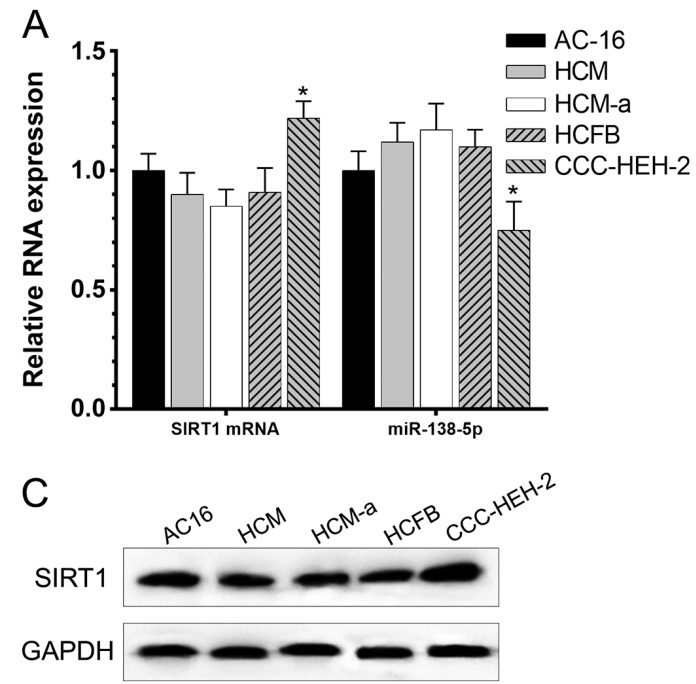

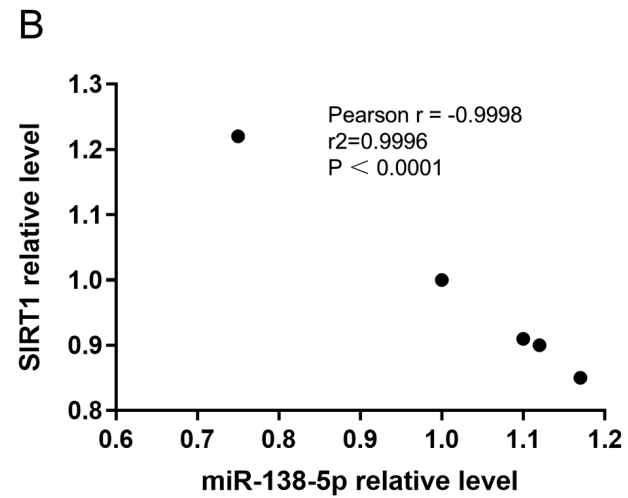

Figure 1. A negative correlation between SIRT1 and miR-138-5p in cardiomyocytes. (A) The relative mRNA level of SIRT1 and miR-138-5p. (B) Negative correlation between SIRT1 and miR-138-5p. (C) The relative level of SIRT1. SIRT1, sirtuin 1; miR, microRNA. "P<0.05 vs. AC-16.

\section{A SIRT1 3'-UTR 5'-GUACAGGAAUUGUUC-CACCAGCA-3' miR-138-5p 3'-GCCGGACUAAGUGUUGUGGUCGA-5'}

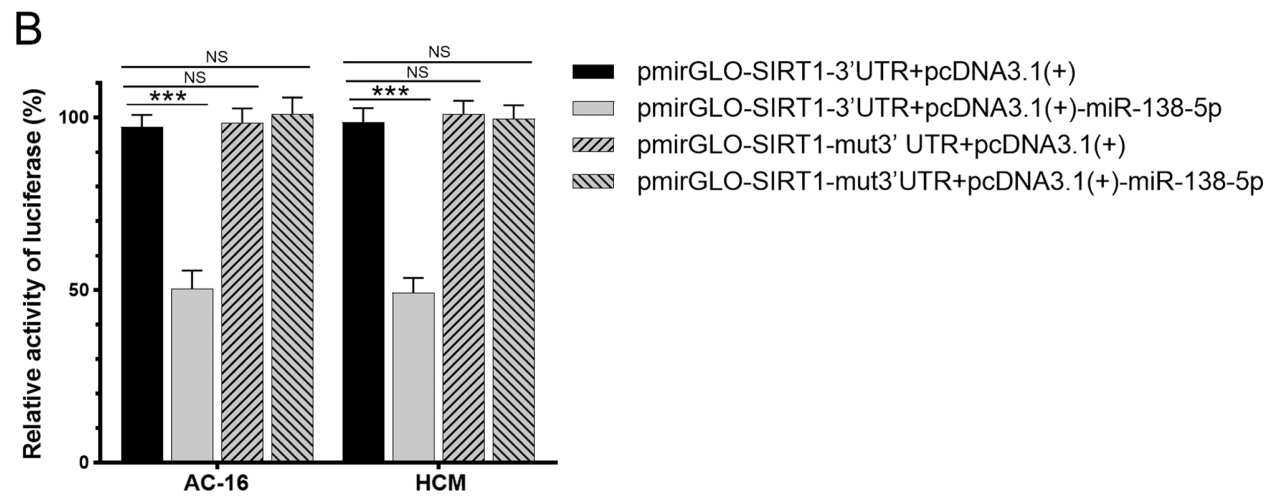

Figure 2. miR-138-5p targets SIRT1. (A) The binding sites in the 3'-UTR of miR-138-5p and SIRT1 were predicted using TargetScan. (B) The direct binding between miR-138-5p and SIRT1 was analyzed by the dual-luciferase reporter assay. ${ }^{* * *} \mathrm{P}<0.001$. miR, microRNA; UTR, untranslated region; SIRT1, sirtuin 1; NS, not significant.

activity of SIRT1 in AC-16 and HCM cells transfected with the miR-138-5p mimic, miR-138-5p inhibitor or negative control were investigated. It was demonstrated that the mimic significantly increased the level of miR-138-5p while the inhibitor decreased the level of miR-138-5p (Fig. 3A); overexpression of miR-138-5p significantly downregulated the mRNA and protein expression of SIRT1 in AC-16 and HCM cells (Fig. 3B and C). Furthermore, the enzymatic activity of SIRT1 was declined in AC-16 and HCM cells overexpressing miR-138-5p. By contrast, knockdown of miR-138-5p significantly upregulated SIRT1 and enhanced its enzyme activity (Fig. 3D). It was demonstrated that miR-138-5p negatively regulated the relative level of SIRT1 and its enzyme activity.

miR-138-5p regulates the HF process via SIRT1-regulated $p 53$ signaling. AC-16 and HCM cells were induced with $200 \mu \mathrm{M}$ $\mathrm{H}_{2} \mathrm{O}_{2}$ for $6 \mathrm{~h}$, and upregulated NPPB and ROS was detected, suggesting successful generation of in vitro $\mathrm{HF}$ models (Fig. 4A and B). Subsequently, AC-16 and HCM cells were transfected with the miR-138-5p mimic, miR-138-5p inhibitor or negative control for $48 \mathrm{~h}$, followed by $\mathrm{H}_{2} \mathrm{O}_{2}$ induction for a further $6 \mathrm{~h}$. These results demonstrated that $\mathrm{H}_{2} \mathrm{O}_{2}$ induction in AC-16 and HCM cells significantly upregulated miR-138-5p. Overexpression of miR-138-5p markedly downregulated SIRT1 and enhanced the rate of acetylated p53 in $\mathrm{H}_{2} \mathrm{O}_{2}$-induced cardiomyocytes (Fig. 4C and D). Expression changes in SIRT1 and acetylated p53 exhibited the opposite trends by knockdown of miR-138-5p. In addition, transfection of the miR-138-5p mimic in $\mathrm{H}_{2} \mathrm{O}_{2}$-induced AC-16 and HCM cells significantly increased the rate of apoptotic cells, while transfection of the miR-138-5p inhibitor decreased the apoptosis rate (Fig. 4E). It was concluded that miR-138-5p inhibited deacetylation of p53 through suppressing the expression level and enzyme activity of SIRT1, thereby regulating the process of HF. 
A

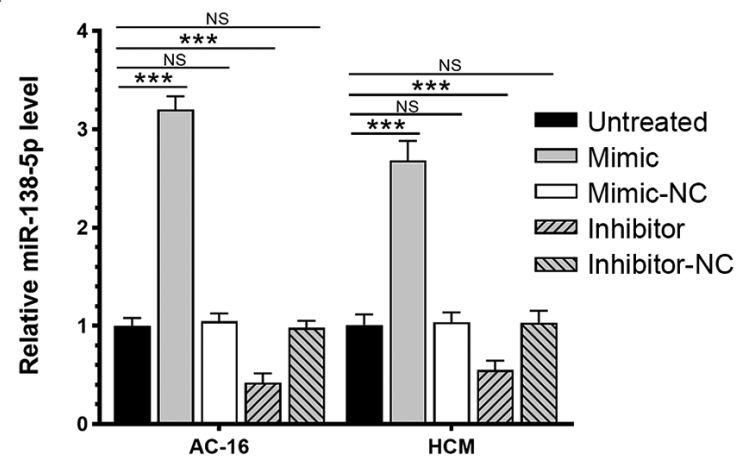

C

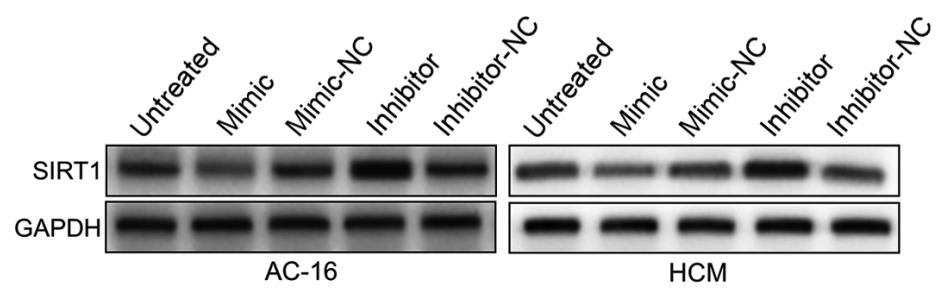

B

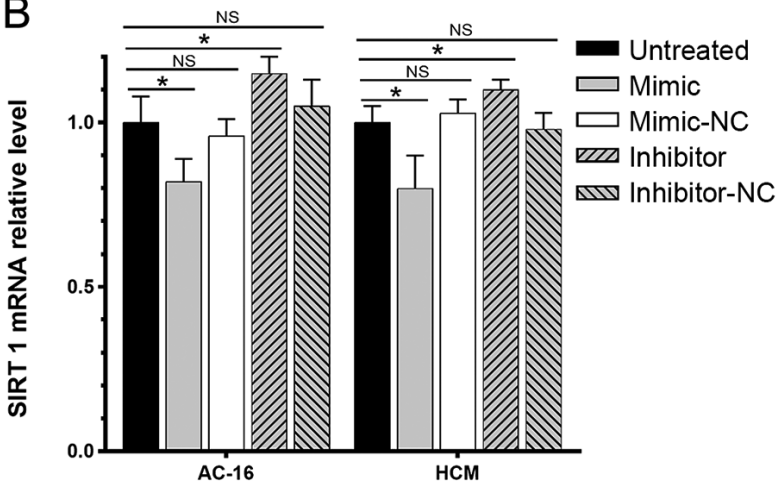

D

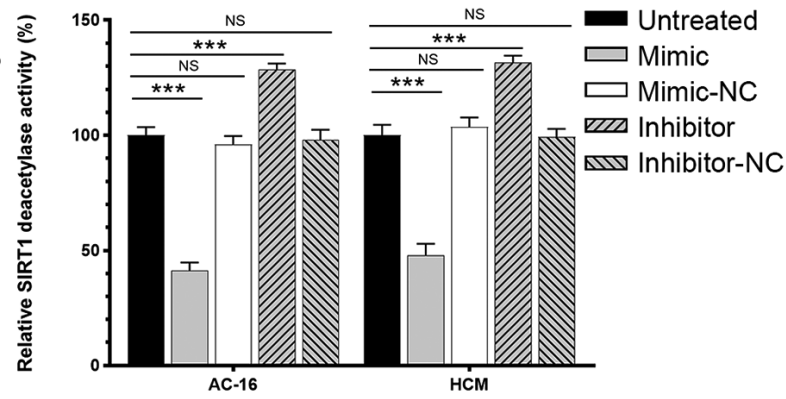

Figure 3. miR-138-5p inhibits the expression level and enzyme activity of SIRT1. (A) The efficacy of miR-138-5p mimic and inhibitor. (B) The relative mRNA expression of SIRT1. (C) The protein expression of SIRT1. (D) The enzyme activity of SIRT1 in AC-16 and HCM cells transfected with the miR-138-5p mimic, mimic NC, miR-138-5p inhibitor, or inhibitor NC, respectively. ${ }^{*} \mathrm{P}<0.05,{ }^{* * *} \mathrm{P}<0.001$. miR, microRNA; SIRT1, sirtuin 1; NC, negative control; NS, not significant.

Protective effect of SIRT1 in HF. In AC-16 and HCM with lentiviral transfection (GV287-SIRT1 or GV287), the mRNA levels of SIRT1 were significantly upregulated (Fig. 5A). These cells were overexpressing SIRT1 when transfected with miR-138-5p mimic or inhibitor, and were then induced with $\mathrm{H}_{2} \mathrm{O}_{2}$ for $6 \mathrm{~h}$ to create an in vitro $\mathrm{HF}$ model. It was demonstrated that the rate of acetylated p53 and the apoptosis rate were significantly decreased in cardiomyocytes overexpressing SIRT1, indicating improved survival of cells (Fig. 5B-D). The results demonstrated that overexpression of SIRT1 may effectively reverse the effect of miR-138-5p mimic or inhibitor, and decrease the acetylation of $\mathrm{p} 53$, thereby protecting cardiomyocytes and decreasing apoptosis in the $\mathrm{H}_{2} \mathrm{O}_{2}$-induced HF model.

\section{Discussion}

$\mathrm{HF}$ is the final outcome of numerous cardiovascular diseases. The incidence and mortality of HF are high, and it endangers the health of patients with cardiovascular and cerebrovascular diseases (18). Myocardial infarction is the main cause of HF (19). It is well known that cardiomyocytes are not renewable. Necrotic cardiomyocytes in the myocardial infarction area are recognized and cleared by the immune system, and they are replaced by scar tissues (20). As a consequence, the structure and function of the remaining myocardium are disrupted, eventually leading to HF (12). The apoptosis of cardiomyocytes often occurs throughout the process of heart disease, and improvement of cardiac function largely relies on the surviving cardiomyocytes $(21,22)$. It has been demonstrated that oxidative stress is the main cause of hypoxic and ischemic heart diseases $(23,24)$. Under pathological conditions, the balance between the production and clearance of ROS is disrupted. Excessive production of ROS results in oxidative stress in tissues. Specifically, the accumulation of ROS and oxygen radicals occurs during the pathological progression of myocardial ischemia, reperfusion and cardiac remodeling, thereby damaging the tissues and causing cell necrosis and apoptosis (25). Piek et al (15) reported that NPPB may be a specific biomarker of HF and Shi et al (16) used NPPB as a biomarker of HF and proved that the sodium-glucose cotransporter 2 inhibitor empagliflozin may be effective for HF. The present study generated in vitro $\mathrm{HF}$ models by $\mathrm{H}_{2} \mathrm{O}_{2}$ induction in AC-16 and HCM cells to mimic oxidative stress damage. The increase in NPPB transcription and MFI of ROS was considered as success of the generated model $(15,16)$.

MiRNAs are a type of non-coding RNA that are 22 nucleotides in length. They are extensively expressed in eukaryotes and participate in post-transcriptional regulation by binding to the 3'-UTR of the target mRNA, thereby degrading them or inhibiting their translation. miRNAs are vital regulators involved in the onset and progression of diseases, including heart diseases. It has been reported that knockdown of miR-21 attenuates proliferation of cardiac fibroblasts and cardiac interstitial fibrosis by downregulating key proteins in the ERK-MAPK signaling, thereby improving cardiac hypertrophy and reversing cardiac remodeling (26). Deficiency of miR-208 prevents cardiac hypertrophy, myocardial fibrosis and cardiac remodeling in overloaded mouse cardiomyocytes (27). Through acting on the expression and secretion of 
A

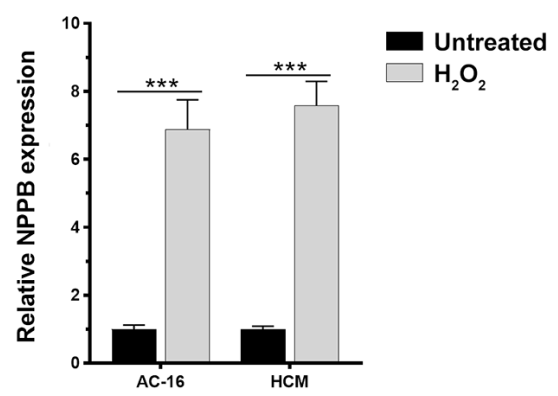

B

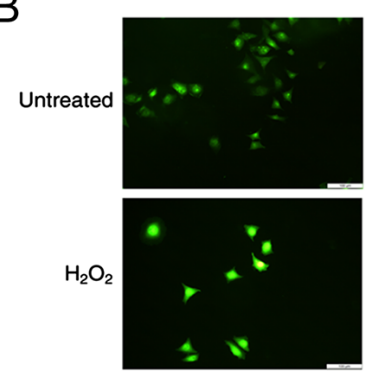

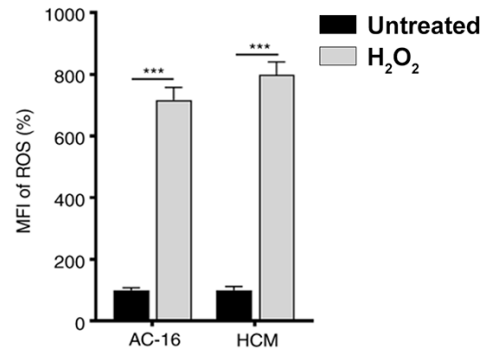
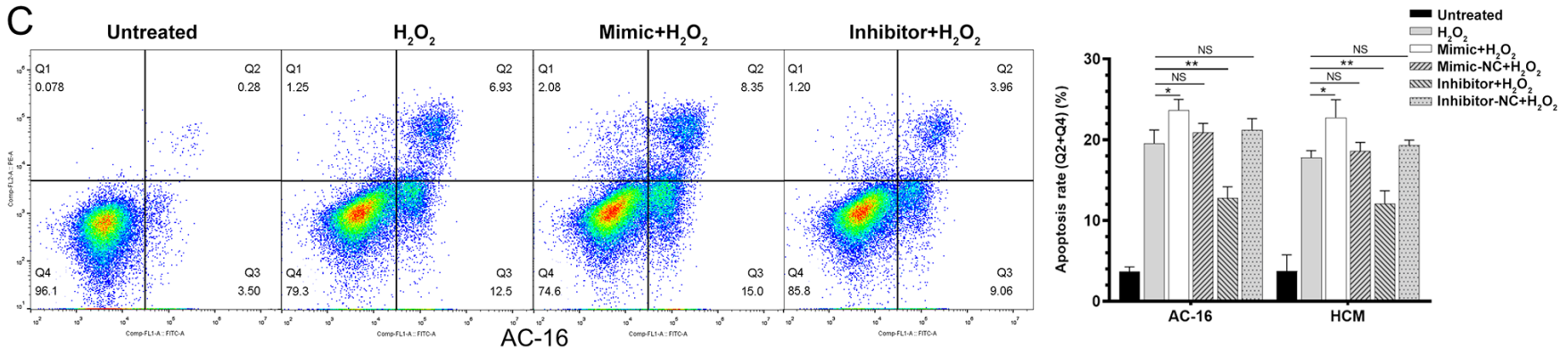

$\mathrm{D}$
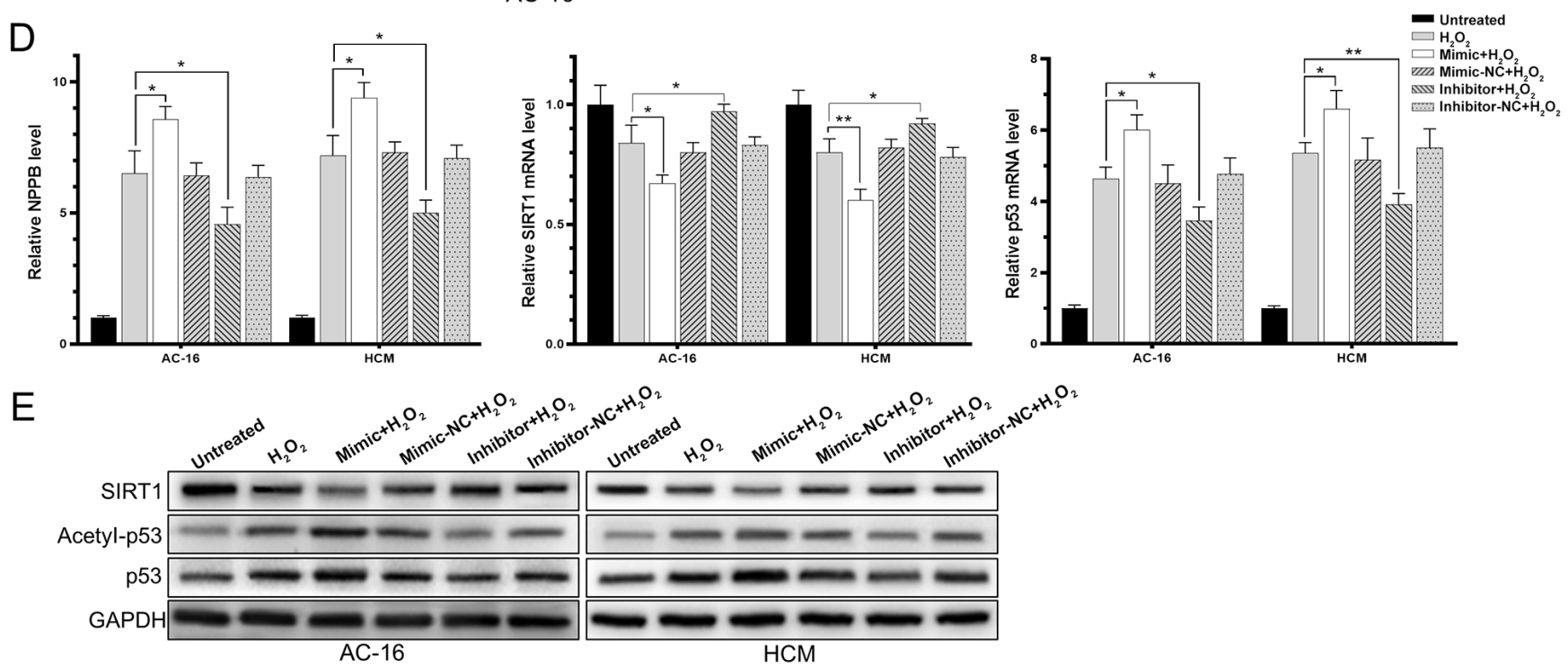

Figure 4. miR-138-5p regulates the heart failure process via SIRT1-regulated 553 signaling. AC-16 and HCM were induced with $\mathrm{H}_{2} \mathrm{O}_{2}$ for $6 \mathrm{~h}$. (A) Upregulated NPPB level. (B) Mean fluorescence intensity of reactive oxygen species. (C) Apoptosis proportion. (D) Relative mRNA levels of SIRT1, p53 and NPPB. (E) Protein levels of SIRT1, p53 and acetyl-p53. ${ }^{*} \mathrm{P}<0.05,{ }^{* *} \mathrm{P}<0.01,{ }^{* * *} \mathrm{P}<0.001$. miR, microRNA; NC, negative control; SIRT1, sirtuin $1 ; \mathrm{H}_{2} \mathrm{O}_{2}$, hydrogen peroxide; NPPB, natriuretic peptide precursor B; NS, not significant.

T cells and upregulating KLF13, miR-147b induces the activation of caspase signaling and, in turn, drives cardiomyocyte apoptosis (25). The inhibitory effect of miR-1 on cardiomyocyte proliferation is abolished by overexpressed miR-195. As a result, hypertrophic and disorderly arranged cardiomyocytes trigger the onset of dilated cardiomyopathy and HF (28). As a tumor suppressor gene, miR-138-5p is conducive to prevent tumor progression and metastasis $(10,29,30)$. Its potential biological function in cardiac diseases has been rarely reported. A latest study revealed that SIRT1 is a potential candidate of target gene binding of miR-138-5p by TargetScan analysis (31). MiR-138-5p is upregulated in insulin-resistant HepG2 cells induced by TNF- $\alpha$, which affects glucose uptake and glycogen synthesis via targeting SIRT1. It is speculated that miR-138-5p is a promising therapeutic target for insulin resistance in the diabetic population (32). Wang et al (10) concluded that overexpression of miR-138-5p deteriorates ischemia-reperfusion injury in the heart by inactivating SIRT1-PGC-1 $\alpha$ signaling. The present study confirmed a negative correlation between miR-138-5p and SIRT1 levels in cardiomyocyte cell lines. Furthermore, the dual-luciferase reporter assay confirmed the ability of miR-138-5p to regulate post-transcriptional translation and enzymatic activity of SIRT1 via binding to its 3'-UTR.

SIRT1 belongs to Class III of the sirtuin family, and its capacity of protein deacetylation is an important post-translational modification. The biological functions of SIRT1 in the process of HF have been previously identified (33). For instance, SIRT1 can modify mitochondrial function and increase ATP production by deacetylating histone and mitochondrial proteins (i.e., UCP-2 and PGC-1 $\alpha$ ), thereby improving cardiomyocyte metabolism and 

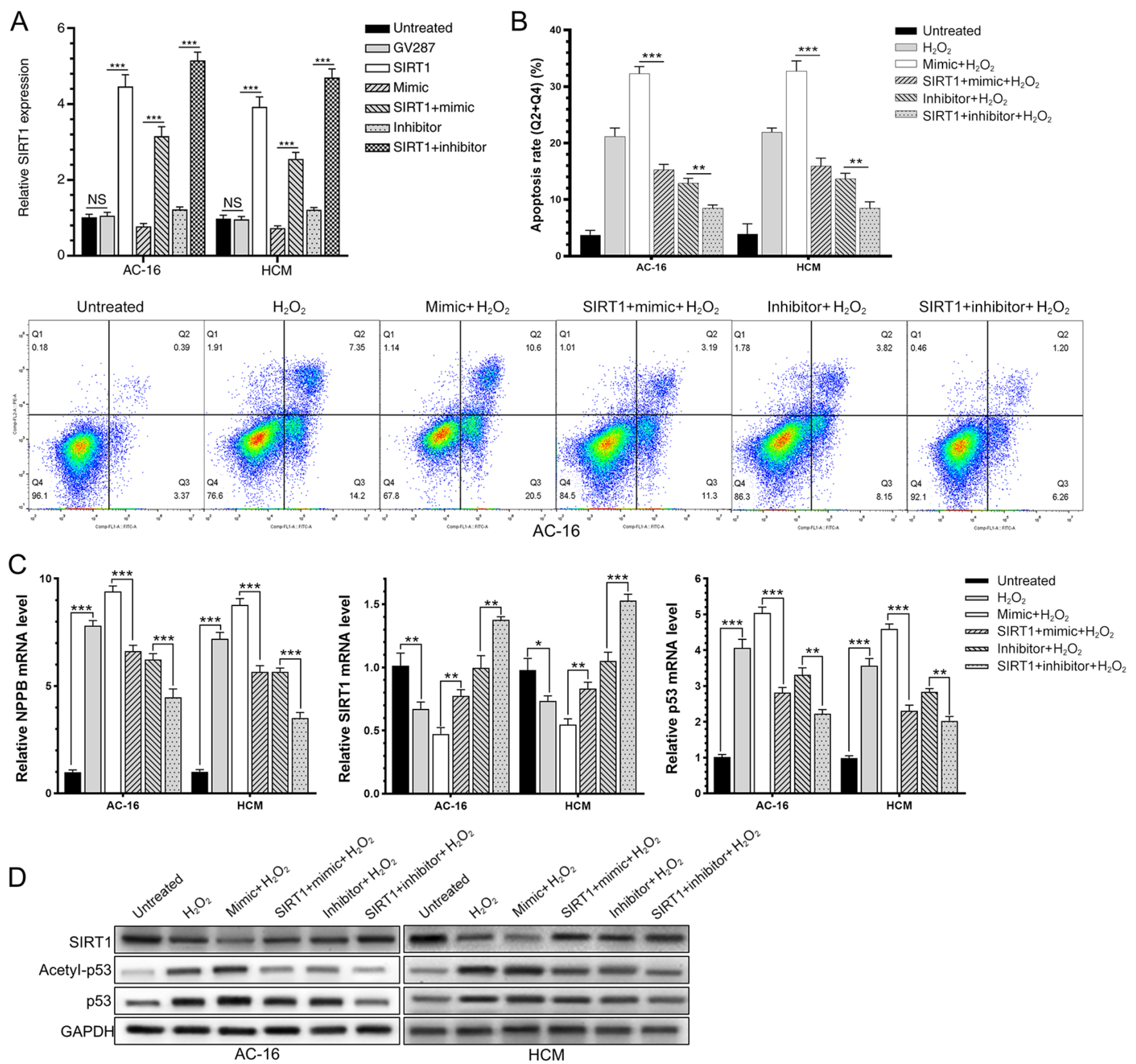

Figure 5. Protective effect of SIRT1 in heart failure. (A) Relative SIRT1 mRNA expression of AC-16 and HCM cells overexpressing SIRT1. (B) Apoptosis in $\mathrm{H}_{2} \mathrm{O}_{2}$-induced AC-16 and HCM cells overexpressing SIRT1. (C) The relative mRNA expression of NPPB, SIRT1 and p53. (D) The protein expression of SIRT1, p53 and acetyl-p53 in $\mathrm{H}_{2} \mathrm{O}_{2}$-induced AC-16 and HCM cells overexpressing SIRT1. ${ }^{*} \mathrm{P}<0.05,{ }^{* *} \mathrm{P}<0.01,{ }^{* * *} \mathrm{P}<0.001$. SIRT1, sirtuin $1 ; \mathrm{H}_{2} \mathrm{O}_{2}$, hydrogen peroxide; NS, not significant.

alleviating the process of HF (7,34). Through deacetylating FOXO1, SIRT1 is responsible for activating Rab7, and thus, it exerts its protective effect on starvation-induced moribund myocardium via blocking apoptosis and clearing damaged mitochondria $(35,36)$. The sarcoplasmic reticulum absorbs calcium through the sarcoplasmic reticulum $\mathrm{Ca}^{2+}$-ATPase (SERCA2a). Dysfunctional SERCA2a may cause a decrease in calcium storage of the sarcoplasmic reticulum. The impaired systolic function of the heart further aggravates HF. Following treatment with resveratrol (SIRT1 activator) in mice with type 1 diabetes mellitus, the abnormally expressed SERCA2a is restored, and notably, mouse ventricular function and ventricular dilation are restored. It has been suggested that SIRT1 improves HF through regulating calcium ions in cardiomyocytes $(37,38)$. In addition, SIRT1 contributes toward clearing mitochondria-generated ROS and relieving symptoms of decreased ejection fraction, myocardial fibrosis and necrosis by upregulating Mn-SOD with the involvement of HIF-2 $\alpha$ (39). The results of the present study illustrated that the upregulated miR-138-5p in the in vitro HF models significantly downregulated the protein expression of SIRT1 and its enzyme activity. Overexpression of miR-138-5p further deteriorated the survival of cardiomyocytes by downregulating SIRT1. By contrast, knockdown of miR-138-5p yielded the opposite result and the survival of cardiomyocytes was significantly improved.

p53 is the first non-histone substrate to be deacetylated by SIRT1, and thus its pro-apoptotic activity is suppressed. 
Sano et al (40) proposed that inactivation of p53 signaling in cardiomyocytes enhances the activity of HIF-1 and induces capillary angiogenesis, which is beneficial in relieving compensatory cardiac hypertrophy. Chen et al (41) identified an interaction between HIF-1 and SIRT1. In a study conducted by Hong et al (42), resveratrol blocked p53 signaling by activating SIRT1 in H9C2 cells, thereby strengthening cardiac function in mice with myocardial infarction. These findings suggest that SIRT1-induced deacetylation of p53 is an important way of preventing HF. The results of the present study demonstrated that in the in vitro HF models, stimulation of apoptosis of cardiomyocytes may be attributed to the decreased translational level of SIRT1 and increased acetylation of p53. Overexpression of miR-138-5p decreased the translational level and enzyme activity of SIRT1, which further activated p53 and aggravated cardiomyocyte apoptosis. By contrast, knockdown of miR-138-5p exerted a protective effect on damaged cardiomyocytes through enhancing SIRT-induced deacetylation of $\mathrm{p} 53$.

In conclusion, the present study identified the upregulation of miR-138-5p at the cellular level in vitro, clarified the targeted association between mir-138-5p and SIRT1, and verified that mir-138-5p may inhibit the post-transcriptional translation process of SIRT1 by targeting SIRT1 mRNA, thereby promoting the process of HF. The present study was based on cellular in vitro research, and more in-depth in vivo research is required.

miR-138-5p is significantly upregulated in the in vitro $\mathrm{HF}$ models. Through inhibiting the translational level and enzyme activity of SIRT1, miR-138-5p induces worsening of HF via activating p53 signaling. miR-138-5p and SIRT1 may be promising diagnostic biomarkers and therapeutic targets for HF.

\section{Acknowledgements}

Not applicable.

\section{Funding}

The present study was supported by the Shenzhen Science and Technology Innovation Committee (grant no. JCYJ20180302173917265).

\section{Availability of data and materials}

The datasets used and/or analyzed during the current study are available from the corresponding author on reasonable request.

\section{Authors' contributions}

SS, CW and JW conceived and designed the study, performed the experiments, and analyzed and interpreted the data. All of the authors agreed to be accountable for all aspects of the work in ensuring that questions related to the accuracy and integrity of any part of the work are appropriately investigated and resolved. All authors confirm the authenticity of all the raw data, and read and approved the final manuscript.

\section{Ethics approval and consent to participate}

Not applicable.

\section{Patient consent for participation}

Not applicable.

\section{Competing interests}

The authors declare that they have no competing interests.

\section{References}

1. Luscher TF: Heart failure: The cardiovascular epidemic of the 21st century. Eur Heart J 36: 395-397, 2015.

2. The L: Heart failure: The need for improved treatment and care. Lancet 392: 451, 2018.

3. Persson H, Linder-Klingsell E, Eriksson SV and Erhardt L: Heart failure after myocardial infarction: The importance of diastolic dysfunction. A prospective clinical and echocardiographic study. Eur Heart J 16: 496-505, 1995.

4. Cech TR and Steitz JA: The noncoding RNA revolution-trashing old rules to forge new ones. Cell 157: 77-94, 2014.

5. D'Onofrio N, Servillo L and Balestrieri ML: SIRT1 and SIRT6 signaling pathways in cardiovascular disease protection. Antioxid Redox Signal 28: 711-732, 2018.

6. Alcendor RR, Gao S, Zhai P, Zablocki D, Holle E, Yu X, Tian B, Wagner T, Vatner SF and Sadoshima J: Sirt1 regulates aging and resistance to oxidative stress in the heart. Circ Res 100: 1512-1521, 2007.

7. Waldman M, Cohen K, Yadin D, Nudelman V, Gorfil D, Laniado-Schwartzman M, Kornwoski R, Aravot D, Abraham NG, Arad M and Hochhauser E: Regulation of diabetic cardiomyopathy by caloric restriction is mediated by intracellular signaling pathways involving 'SIRT1 and PGC-1alpha'. Cardiovase Diabetol 17: 111, 2018.

8. Yang H, Bi Y, Xue L, Wang J, Lu Y, Zhang Z, Chen X, Chu Y, Yang R, Wang R and Liu G: Multifaceted modulation of SIRT1 in cancer and inflammation. Crit Rev Oncog 20: 49-64, 2015.

9. Dong G, Wang B, An Y, Li J, Wang X, Jia J and Yang Q: SIRT1 suppresses the migration and invasion of gastric cancer by regulating ARHGAP5 expression. Cell Death Dis 9: 977, 2018.

10. Wang C, Sun X, Qiu Z and Chen A: MiR-138-5p exacerbates hypoxia/reperfusion-induced heart injury through the inactivation of SIRT1-PGC-1alpha. Inflamm Res 68: 867-876, 2019.

11. Yang J, Yang XS, Zhang Q, Zhuang X, Dong XK, Jiang YH, Tao YN and Yang CH: Downregulated LINC01614 ameliorates Hypoxia/reoxygenation-stimulated myocardial injury by directly sponging microRNA-138-5p. Dose Response 18: $1559325820913786,2020$.

12. Minicucci MF, Azevedo PS, Polegato BF, Paiva SA and Zornoff LA: Heart failure after myocardial infarction: Clinical implications and treatment. Clin Cardiol 34: 410-414, 2011.

13. Zhang L, Wang YN, Ju JM, Shabanova A, Li Y, Fang RN, Sun JB, Guo YY, Jin TZ, Liu YY, et al: Mzb1 protects against myocardial infarction injury in mice via modulating mitochondrial function and alleviating inflammation. Acta Pharmacol Sinica: Aug 5, 2020 doi: 10.1038/s41401-020-0489-0 (Epub ahead of print).

14. Wei Q, Zhou HY, Shi XD, Cao HY and Qin L: Long noncoding RNA NEAT1 promotes myocardiocyte apoptosis and suppresses proliferation through regulation of miR-129-5p. J Cardiovasc Pharmacol 74: 535-541, 2019.

15. Piek A, Suthahar N, Voors AA, de Boer RA and Sillje HHW: A combined bioinformatics, experimental and clinical approach to identify novel cardiac specific heart failure biomarkers: Is Dickkopf-3 (DKK3) a possible candidate? Eur J Heart Fail 22: 2065-2074, 2020.

16. Shi X, Verma S, Yun J, Brand-Arzamendi K, Singh KK, Liu X, Garg A, Quan A and Wen XY: Effect of empagliflozin on cardiac biomarkers in a zebrafish model of heart failure: Clues to the EMPA-REG OUTCOME trial? Mol Cell Biochem 433: 97-102, 2017.

17. Livak KJ and Schmittgen TD. Analysis of relative gene expression data using real-time quantitative PCR and the 2(-Delta Delta C(T)) method. Methods 25: 402-408, 2001.

18. Lelonek M, Stopczynska I, Koroscik E, Straburzynska-Migaj E and Gruchala M: Multicenter experiences with levosimendan therapy and its safety in patients with decompensated advanced heart failure. Adv Clin Exp Med 29: 1305-1312, 2020. 
19. Mia MM, Cibi DM, Abdul Ghani SAB, Song W, Tee N, Ghosh S, Mao J, Olson EN and Singh MK: YAP/TAZ deficiency reprograms macrophage phenotype and improves infarct healing and cardiac function after myocardial infarction. PLoS Biol 18: e3000941, 2020

20. Li J, Jia L, Hao Z, Xu Y, Shen J, Ma C, Wu J, Zhao T, Zhi Y, Li P, et al: Site-specific N-glycoproteomic analysis reveals upregulated sialylation and core fucosylation during transient regeneration loss in neonatal mouse hearts. J Proteome Res 19: 3191-3200, 2020.

21. Badreddin A, Fady Y, Attia H, Hafez M, Khairallah A, Johar D and Bernstein L: What role does the stress response have in congestive heart failure? J Cell Physiol 233: 2863-2870, 2018.

22. Szobi A, Gonçalvesová E, Varga ZV, Leszek P, Kuśmierczyk M, Hulman M, Kyselovič J, Ferdinandy P and Adameová A: Analysis of necroptotic proteins in failing human hearts. J Transl Med 15: $86,2017$.

23. Ravingerová $T$, Čarnická $S$, Nemčeková M, Ledvényiová V, Adameová A, Khandelwal VK, Zálešák $M$ and Kolář F: The impact of lifestyle-related risk factors on cardiac response to ischemia and possibilities to restore impaired ischemic tolerance. Physiol Res 61 (Suppl 2): S1-S10, 2012.

24. Turer AT and Hill JA: Pathogenesis of myocardial ischemia-reperfusion injury and rationale for therapy. Am J Cardiol 106: 360-368, 2010

25. Gu M, Wang J, Wang Y, Xu Y, Zhang Y, Wu W and Liao S: miR-147b inhibits cell viability and promotes apoptosis of rat H9c2 cardiomyocytes via down-regulating KLF13 expression. Acta Biochim Biophys Sin (Shanghai) 50: 288-297, 2018.

26. Adam O, Lohfelm B, Thum T, Gupta SK, Puhl SL, Schafers HJ, Böhm M and Laufs U: Role of miR-21 in the pathogenesis of atrial fibrosis. Basic Res Cardiol 107: 278, 2012.

27. Satoh M, Minami Y, Takahashi Y, Tabuchi T and Nakamura M: Expression of microRNA-208 is associated with adverse clinical outcomes in human dilated cardiomyopathy. J Card Fail 16: 404-410, 2010

28. van Rooij E, Sutherland LB, Liu N, Williams AH, McAnally J, Gerard RD, Richardson JA and Olson EN: A signature pattern of stress-responsive microRNAs that can evoke cardiac hypertrophy and heart failure. Proc Natl Acad Sci USA 103: 18255-18260, 2006

29. Zhu D, Gu L, Li Z, Jin W, Lu Q and Ren T: miR-138-5p suppresses lung adenocarcinoma cell epithelial-mesenchymal transition, proliferation and metastasis by targeting ZEB2. Pathol Res Pract 215: 861-872, 2019

30. Wang X, Zhao Y, Cao W, Wang C, Sun B, Chen J, Li S, Chen J, Cui M, Zhang B, et al: miR-138-5p acts as a tumor suppressor by targeting hTERT in human colorectal cancer. Int J Clin Exp Pathol 10: 11516-11525, 2017.

31. Ma J, Zhang Y, Ji H, Chen L, Chen T, Guo C, Zhang S, Jia J and Niu P: Overexpression of miR-138-5p suppresses $\mathrm{MnCl}_{2}$-induced autophagy by targeting SIRT1 in SH-SY5Y cells. Environ Toxicol 34: 539-547, 2019.
32. Luan B and Sun C: MiR-138-5p affects insulin resistance to regulate type 2 diabetes progression through inducing autophagy in HepG2 cells by regulating SIRT1. Nutr Res 59: 90-98, 2018.

33. Hsu CP, Odewale I, Alcendor RR and Sadoshima J: Sirt1 protects the heart from aging and stress. Biol Chem 389: 221-231, 2008.

34. Kutsche HS, Schreckenberg R, Weber M, Hirschhauser C, Rohrbach S, Li L, Niemann B, Schulz R and Schlüter KD: Alterations in glucose metabolism during the transition to heart failure: The contribution of UCP-2. Cells 9: 552, 2020.

35. Hariharan N, Maejima Y, Nakae J, Paik J, Depinho RA and Sadoshima J: Deacetylation of FoxO by Sirt1 plays an essential role in mediating starvation-induced autophagy in cardiac myocytes. Circ Res 107: 1470-1482, 2010.

36. Gottlieb RA, Finley KD and Mentzer RM Jr: Cardioprotection requires taking out the trash. Basic Res Cardiol 104: 169-180, 2009.

37. Sulaiman M, Matta MJ, Sunderesan NR, Gupta MP, Periasamy M and Gupta M: Resveratrol, an activator of SIRT1, upregulates sarcoplasmic calcium ATPase and improves cardiac function in diabetic cardiomyopathy. Am J Physiol Heart Circ Physiol 298: H833-H843, 2010.

38. Chen X, Zhang X, Gross S, Houser SR and Soboloff J: Acetylation of SERCA2a, another target for heart failure treatment? Circ Res 124: 1285-1287, 2019

39. Dioum EM, Chen R, Alexander MS, Zhang Q, Hogg RT, Gerard RD and Garcia JA: Regulation of hypoxia-inducible factor 2alpha signaling by the stress-responsive deacetylase sirtuin 1. Science 324: 1289-1293, 2009.

40. Sano M, Minamino T, Toko H, Miyauchi H, Orimo M, Qin Y, Akazawa H, Tateno K, Kayama Y, Harada M, et al: p53-induced inhibition of Hif-1 causes cardiac dysfunction during pressure overload. Nature 446: 444-448, 2007.

41. Chen R, Dioum EM, Hogg RT, Gerard RD and Garcia JA: Hypoxia increases sirtuin 1 expression in a hypoxia-inducible factor-dependent manner. J Biol Chem 286: 13869-13878, 2011.

42. Hong W, Tatsuo S, Shou-Dong W, Qian Z, Jian-Feng H, Jue W, Chen J, Hai-Yan Q and Yue-Jin Y: Resveratrol upregulates cardiac SDF-1 in mice with acute myocardial infarction through the deacetylation of cardiac p53. PLoS One 10: e0128978, 2015.

This work is licensed under a Creative Commons Attribution-NonCommercial-NoDerivatives 4.0 International (CC BY-NC-ND 4.0) License. 